\title{
Modelos para el diagnóstico y cuantificación DEL PODER DE MERCADO EN SECTORES INDUSTRIALES: UN ENFOQUE DE ESTRUCTURA-CONDUCTA-RESULTADOS
}

\author{
José Benjamín Gallego Alzate ${ }^{1}$
}

\section{Resumen}

Este artículo tiene como propósito presentar dos modelos para diagnosticar y cuantificar el poder de mercado en sectores industriales, inscriptos en el paradigma clásico "Estructura-Conducta-Resultados". En general, se muestra como el poder de mercado está estructuralmente relacionado con los índices de concentración o cuota de mercado, la elasticidad precio de la demanda, la variación conjetural y los rendimientos a escala.

\section{Palabras Claves:}

Oligopolio, poder de mercado, índice de Lerner, Concentración.

\section{Abstract}

In this paper, two models about diagnosis and quantification the power monopoly in industrial sector is presented. These models are part of the classic paradigm "Structure- Conduct- Performance". In general, the power market is structurally related with concentration

1 Economista Agrícola y Magister en Ciencias Económicas de la Universidad Nacional de Colombia. Especialista en Política Económica de la Universidad de Antioquia, y en Docencia Universitaria de la Universidad Industrial de Santander. Director del Grupo de Investigación en Gestión Tecnológica. Profesor Asociado del InstituTo Tecnológico Metropolitano.

E-mail: josegallego@itm.edu.co 
indexes or market share, the price elasticity of demand, the conjectural variation and returns to scale.

\section{Key Words:}

Oligopoly, Market power, Lerner index, Concentration. 


\section{INTRODUCCIÓN}

El propósito de este texto es presentar la revisión de algunos modelos teóricos que permitan diagnosticar y cuantificar, con su aplicación empírica, la existencia de poder de mercado en los diferentes sectores industriales. La revisión cubre modelos en el marco del paradigma clásico "Estructura- Conducta- Resultados" y su desarrollo es básicamente matemático.

$\mathrm{El}$ artículo surge del avance investigativo del proyecto "El poder de mercado en las industrias basadas en la ciencia: los casos de las industrias Química y Farmacéutica”, en el que se asume la clasificación de los sectores industriales realizada por Keith Pavitt (1984). Para Pavitt, las industrias basadas en la ciencia son: Química, Biotecnología, Farmacéutica y Electrónica. En estas industrias, la tecnología empleada en los procesos productivos es propias, el origen de las innovaciones es desde la investigación y el desarrollo (I+D) y la aplicación de conocimientos de centros públicos, y son protegidas por medio de patentes y secretos industriales; el tamaño de las empresas es grande, y la innovación de productos y procesos procura la reducción de costos y el aumento de la prestaciones de servicios.

Se entiende por poder de mercado, la habilidad de una firma o grupo de firmas para establecer el precio de los bienes sobre el nivel de la competencia, durante un periodo de tiempo ${ }^{2}$. Este poder permite obtener beneficios sobre los niveles de competencia perfecta, denominados rentas monopolísticas ${ }^{3}$. Desde un punto de vista microeconómico, el poder de mercado significa reducción de la competencia, pérdida irrecuperable de eficiencia y transferencia de excedente del consumidor al productor.

2 AMERICAN BAR ASSOCIATION (2005). Market Power Handbook. Competition Law and Economic Foundations. Chicago: ABA Publishing. pp1-2

3 STIGLITZ, Joseph E. (1998). Microeconomía. España: Ariel. p 353 


\section{Modelo de OLIGopolio. Índice DE LeRner Y CONCENTRACIÓN DE MERCADO}

El oligopolio es una estructura de mercado compuesto por más de una empresa, pero pocas, de manera que el comportamiento de cualquiera de ellas tiene efecto sobre el precio; por ello, en el modelo de oligopolio se enfatiza en la interdependencia del comportamiento del rival. Las empresas actúan independientemente o bajo algún grado de colusión; y basan sus decisiones de precios y producción fundamentadas en la acción esperada del rival a tales decisiones. Las respuestas se mide por medio de la variación conjetural de precios (modelos Edgeworth y de la demanda quebrada) y de producción (modelo Cournot de empresa seguidora y modelo Stackelberg de empresa seguidora o líder).

\subsection{El margen precio - costo marginal o índice de Lerner para una empresa}

En oligopolio y siguiendo un escenario de Cournot, el problema de la empresa $i$ es maximizar el beneficio $\pi_{i}$, que se define como $\pi i=P(X) x_{i}-C\left(x_{i}\right)(1)$ y en el que:

$P(X)=$ Es la función inversa de demanda

$X=$ Es la producción total de la industria, e igual a $\sum_{i=1}^{n} x_{i}$

$x_{i} \quad=$ Producción de la empresa $i$

$X_{-i}=$ Es la producción conjunta de las $n$ empresas del mercado menos la producción de la empresa $i$. Es decir $X_{-i}=X-x_{i}$

Tomando la derivada a la ecuación del beneficio tenemos: 
$\frac{\partial \pi_{i}}{\partial x_{i}}=P(X)+x_{i} \frac{\partial P(X)}{\partial x_{i}}-\frac{\partial c\left(x_{i}\right)}{\partial x_{i}}$. Pero como la derivada de $\frac{\partial P(X)}{\partial x_{i}}$ es igual a $\frac{\partial P(X)}{\partial X} \frac{\partial(X)}{\partial x_{i}}$, esto es $\frac{\partial P(X)}{\partial X}\left(\frac{\partial x_{i}}{\partial x_{i}}+\frac{\partial X_{-i}}{\partial x_{i}}\right)$, y si adicionalmente hacemos $\frac{\partial c\left(x_{i}\right)}{\partial x_{i}}=c^{\prime}($ costo marginal), tenemos que la derivada es entonces: $\frac{\partial \pi_{i}}{\partial x_{i}}=P(X)+x_{i} \frac{\partial P(X)}{\partial X}\left(\frac{\partial x_{i}}{\partial x_{i}}+\frac{\partial X_{-i}}{\partial x_{i}}\right)-c^{\prime}$. Igualando a cero la derivada, tenemos $P(X)+x_{i} \frac{\partial P(X)}{\partial X}\left(1+\frac{\partial X_{-i}}{\partial x_{i}}\right)=c^{\prime}$, siendo $\frac{\partial X_{-i}}{\partial x_{i}}$ la variación conjetural $\lambda_{i}$, esto es, el cambio en la producción conjunta de las $n$ empresas como respuesta a un cambio en la producción de la empresa $i$ (conjetura en cantidades, no en precios).

Bajo un modelo de oligopolio de Cournot, cuyo supuesto central consiste en que cada empresa actúa considerando que la producción de la empresa rival es fija (lo que hace que el término de la variación conjetural de la producción sea cero para las empresas, esto es $\lambda_{i}=0$ ), la condición de máximo beneficio, definida como ingreso marginal igual al costo marginal, es: $P(X)+x_{i} \frac{\partial P(X)}{\partial X}=c^{\prime}$.

Y si denotamos la derivada $\frac{\partial P(X)}{\partial X}=P^{\prime}$, entonces la condición de equilibrio queda expresada como: $P(X)+x_{i} P^{\prime}=c^{\prime}$, o también $P+x_{i} P^{\prime}=c^{\prime}$.

Ahora, si asumimos que la variación conjetural es, en vez de cero, una constante (o sea $\lambda_{i}=\lambda$ ), entones la condición de máximo beneficio para la empresa $i$ es ahora $P+x_{i} P^{\prime}\left(1+\lambda_{i}\right)=c^{\prime}$

Para obtener una medida del grado de poder de mercado, como es el índice de Lerner o margen precio - costo marginal, procedemos de la siguiente manera:

Si a la expresión $P-c^{\prime}=-x_{i} P^{\prime}(1+\lambda)$ la dividimos por $P$ tenemos el margen del precio sobre el costo marginal o índice de Lerner de la empresa $i$ como: $\frac{P-c^{\prime}}{P}=-\frac{x_{i} P^{\prime}\left(1+\lambda_{i}\right)}{P}$; es decir $B_{i}=-\frac{x_{i} P^{\prime}\left(1+\lambda_{i}\right)}{P}$. Si multiplicamos y dividimos el numerador de esta última expresión 
por $X$, y si definimos a $S_{i}=\frac{x_{i}}{X}$, siendo $S_{i}$ la cuota o participación en el mercado de la empresa $i$ (o índice de concentración), y a $\varepsilon_{p x}$ como la elasticidad precio de la demanda $=-\frac{P}{P^{\prime} X}$, entonces:

$$
B_{i}=\frac{\frac{x_{i}}{X} X P^{\prime}\left(1+\lambda_{i}\right)}{P}, \text { es decir } \quad B_{i}=\frac{\frac{x_{i} P^{\prime}(1+\lambda)}{X}}{\frac{P}{X}}=\frac{\frac{x_{i}}{X}(1+\lambda)}{\frac{P}{P^{\prime} X}}
$$

Por lo tanto, el índice de Lerner o de poder de mercado para la empresa $i$ es. $B_{i}=\frac{S_{i}\left(1+\lambda_{i}\right)}{\varepsilon_{P X}}$

Esta última ecuación permite concluir, bajo el escenario descripto, que el poder de mercado de la empresa $i$ depende de su cuota de mercado, de la elasticidad precio de la demanda del bien $X$ y de la expectativa (conjetura constante) de la empresa $i$ sobre la conducta de las empresas rivales.

\subsection{El Índice de Lerner para la industria}

El índice de Lerner para la industria, representado por $B$, se obtiene como la suma de los índices individuales $B_{i}$, con $i=1 \ldots n$, ponderado cada $B_{i}$ por la participación relativa de la empresa $i$ en el mercado. Es decir, $B=\sum_{i=1}^{n} B_{i} * S_{i}$;

esto es $B=\sum_{i=1}^{n}\left[\frac{S_{i}\left(1+\lambda_{i}\right)}{\varepsilon_{P X}}\right] S_{i}$.

Si desarrollando esta última ecuación, tenemos:

$B=\sum_{i=1}^{n}\left[\frac{S_{i}+S_{i} \lambda_{i}}{\varepsilon_{P X}}\right] S_{i}$, o sea $B=\sum_{i=1}^{n} \frac{S_{i}^{2}+S_{i}^{2} \lambda_{i}}{\varepsilon_{P X}}$, luego $B=\sum_{i=1}^{n} \frac{S_{i}^{2}}{\varepsilon_{P X}}+\lambda_{i} \sum_{i=1}^{n} \frac{S_{i}^{2}}{\varepsilon_{P X}}$.

Pero como $\sum_{i=1}^{n} S_{i}{ }^{2}=H$ es el índice de concentración de Herfindahl, la expresión anterior es equivalente a $B=\frac{H}{\varepsilon_{P X}}+\frac{\lambda_{i} H}{\varepsilon_{P X}}$ o sea $B=\frac{\left(1+\lambda_{i}\right) H}{\varepsilon_{P X}}$. 
Si las empresas en el mercado del bien $X$ son tomadoras de precio, el índice de Lerner de la industria es cero $(B=0)$; en cambio si $B=1$, el mercado de dicho bien es un monopolio. Un $B>0$ indica presencia de poder de mercado en la industria, es decir, capacidad de las empresas para determinar el precio de mercado sobre el nivel de competencia.

Sea ahora $\varepsilon_{i}$ la elasticidad conjetural de la empresa $i$, es decir: $\varepsilon_{i}=\frac{\frac{\partial X_{-i}}{X_{-i}}}{\frac{\partial x_{i}}{x_{i}}}$, esto es $\varepsilon_{i}=\frac{\partial X_{-i}}{\partial x_{i}} \frac{x_{i}}{X_{-i}}$ y si suponemos que es constante, el índice de Lerner para la empresa $i, B_{i}=\frac{S_{i}\left(1+\lambda_{i}\right)}{\varepsilon_{P X}}$, se convierte en $B_{i}=\frac{S_{i}+\left(1-S_{i}\right) \varepsilon_{i}}{\varepsilon_{P X}}$. La deducción es la siguiente:

Como $B_{i}=\frac{S_{i}\left(1+\lambda_{i}\right)}{\varepsilon_{P X}}$, es decir $B_{i}=\frac{S_{i}+S_{i} \lambda_{i}}{\varepsilon_{P X}}$, debemos demostrar realmente que $S_{i} \lambda_{i}=\left(1-S_{i}\right) \varepsilon_{i}$.

Recordando que $\lambda_{i}=\frac{\partial X_{-i}}{\partial x_{i}}$, tenemos entonces que $S_{i} \frac{\partial X_{-i}}{\partial x_{i}}$, si multiplicamos y dividimos lo anterior por $X_{i}$ obtenemos $S_{i}\left[\frac{\partial X_{-i}}{\partial x_{i}} \frac{X_{-i}}{X_{-i}}\right]$, pero como $X_{-i}=X-x_{i}$ por ello $S_{i}\left[\frac{\partial X_{-i}\left(X-x_{i}\right)}{X_{-i} \partial x_{i}}\right]$, así $S_{i}\left[\frac{\partial X_{-i} X-\partial X_{-i} x_{i}}{X_{-i} \partial x_{i}}\right]$; o sea $S_{i}\left[\frac{\partial X_{-i} X}{X_{-i} \partial x_{i}}-\frac{\partial X_{-i}}{X_{-i}} \frac{x_{i}}{\partial x_{i}}\right]$. Adicionalmente, si $S_{i}=\frac{x_{i}}{X}$ tenemos entonces que $\frac{x_{i}}{X} \frac{\partial X_{-i} X}{X_{-i} \partial x_{i}}-\frac{x_{i}}{X} \frac{\partial X_{-i}}{X_{-i}} \frac{x_{i}}{\partial x_{i}}$, que cancelando $X$ en la primera parte y reordenando obtenemos: $\frac{\partial X_{-i}}{\partial x_{i}} \frac{x_{i}}{X_{-i}}-\frac{x_{i}}{X} \frac{\partial X_{-i}}{\partial x_{i}} \frac{x_{i}}{X_{-i}}$ (2). Ahora bien, como por definición la elasticidad conjetural de la empresa $i$ es $\varepsilon_{i}=\frac{\partial X_{-i}}{\partial x_{i}} \frac{x_{i}}{X_{-i}}$ y además $S_{i}=\frac{x_{i}}{X}$, al reemplazar estas definiciones en la expresión (2), obtenemos $\varepsilon_{i}-\varepsilon_{i} S_{i}$, esto es $\left(1-S_{i}\right) \varepsilon_{i}$. Así entonces, concluimos que $S_{i} \lambda_{i}=\left(1-S_{i}\right) \varepsilon_{i}$. 


\subsection{Mercado dominado por un grupo de empresas: El índice de Lerner para la empresa y la industria}

Supongamos ahora que la industria del bien $X$ es dominada por un grupo $d$ de empresa y el resto de empresas que conforman la industria, $n-d$, son tomadoras de precio, es decir su $B_{i}=0$. El grupo dominante determina el nivel de producción que maximiza el beneficio.

Sea $X_{d}=\sum^{d} x_{i}$, la producción del grupo de empresas dominantes en la industriàa. Definimos además a $X_{t}=X-X_{d}$, como la producción de las restantes empresas de la industria, el producto del grupo de empresas tomadoras de precio. Así tenemos que ahora $X=X_{d}+X_{t}$.

La función de beneficio a maximizar ahora es

$$
\pi_{d}=P\left(X_{d}+X_{t}\right) X_{d}-\sum_{i=1}^{d} c_{i}\left(x_{i}\right) \text { tomando derivada igualada a cero }
$$

tenemos: $\frac{\partial \pi_{d}}{\partial X_{d}}=P\left(X_{d}+X_{t}\right)+X_{d} \frac{\partial P\left(X_{d}+X_{t}\right)}{\partial X} \frac{\partial X}{\partial X_{d}}-c^{\prime}{ }_{i} x_{i}=0$. O lo que es los mismo: $P X+X_{d} \frac{\partial P(X)}{\partial X}\left(\frac{\partial X_{d}}{\partial X_{d}} \frac{\partial X_{t}}{\partial X_{d}}\right)=c_{i}\left(x_{i}\right)$.

Ahora bien, como: $\left(\frac{\partial X_{d}}{\partial X_{d}}\right)=1 ; \frac{\partial P(X)}{\partial X}=P^{\prime}, \mathrm{y}\left(\frac{\partial X_{t}}{\partial X_{d}}\right)=\left(\lambda_{d t}\right)$,

es decir la variación conjetural de las empresas dominantes respecto al resto de empresas de la industria, la expresión anterior es igual a: $=c_{i}^{\prime}\left(x_{i}\right)$.

Si suponemos además que el grupo de empresas líderes tienen un mismo costo marginal, esto es $c_{i}^{\prime}\left(x_{i}\right)=c_{d}^{\prime}$, entonces la condición de máximo beneficio es $P(X)+X_{d} P^{\prime}\left(1+\lambda_{d t}\right)=c_{d}^{\prime}$.

Bajo este modelo, el índice de Lerner se expresa como:

$B_{d}=\frac{P-c_{d}^{\prime}}{P}=-\frac{X_{d}-P^{\prime}\left(1+\lambda_{d t}\right)}{P}$. Si de nuevo multiplicamos y dividimos el numerador del lado derecho de la igualdad por $X$, entonces $B_{d}=-\frac{\frac{X_{d}}{X} P^{\prime} X\left(1+\lambda_{d t}\right)}{P}$, ahora $S_{d}=\frac{X_{d}}{X}$ es la participación 
en el mercado del grupo de empresas dominantes ${ }^{4}$, tenemos: $B_{d}=\frac{S_{d}\left(1+\lambda_{d t}\right)}{\frac{P}{P^{\prime} X}}$, y como $\frac{P}{P^{\prime} X}$ es la elasticidad precio de la demanda del mercado, el índice de Lerner para el grupo de empresas dominantes es $B_{d}=\frac{S_{d}\left(1+\lambda_{d t}\right)}{\varepsilon_{P X}}$.

Ahora, el índice de poder de mercado promedio de la industria, ponderando por la participación relativa en el mercado $S_{d}$ es: $B=S_{d} B_{d}$.

$$
B=\frac{S_{d} S_{d}\left(1+\lambda_{d t}\right)}{\varepsilon_{P X}}=\frac{S^{2}{ }_{d}\left(1+\lambda_{d t}\right)}{\varepsilon_{P X}} \text {, recordando que el grupo }(n-d)
$$
empresas restantes son tomadoras de precio y su $B_{t}=0^{5}$.

\subsection{Mercado dominado por un grupo de empresas y variación conjetural constante}

En el caso de la industria, sea $\varepsilon_{d t}$ la elasticidad conjetural del grupo dominante determinada como $\frac{\partial X_{t}}{\partial X_{d}} \frac{X_{d}}{X_{t}}$. Supongamos que el grupo de empresas dominantes considera la elasticidad conjetural constantes, entonces el índice Lerner promedio de la industria es ahora $B=\frac{S_{d}^{2}\left(1+\lambda_{d t}\right)}{\varepsilon_{P X}}=\frac{\left(1-\lambda_{d t}\right) S^{2}{ }_{d}+\varepsilon_{d t} S_{d}}{\varepsilon_{P X}}$.

El procedimiento es el siguiente: debemos demostrar que los numeradores son equivalentes; recordando que $S_{d}=\frac{X_{t}}{X}$ y que $\lambda_{d t}=\frac{\partial X_{t}}{\partial X_{d}}$.

4 Pero $\frac{X_{d}}{X}=\sum_{i=1}^{d} S_{i}$, y representa el índice de concentración del grupo de empresas dominantes.

$5 \quad B_{d}$ es el índice de Lerner para el grupo de empresas dominantes, por tener una participación $S_{d}$ de mercado. La participación de las restantes empresas tomadoras de precio es $\left(1-S_{d}\right)$. Ahora, el índice de la industria, al definir $B_{t}$ como el índice de poder de mercado de las empresas tomadoras de precios, es: $B=S_{d} * B_{d}+\left(1-S_{d}\right) B_{t}$, pero como el $B_{t}=0$, entonces $B=S_{d} B_{d}$. 
Tenemos entonces que $S_{d}^{2}\left(1+\lambda_{d t}\right)$ es igual a $S_{d}^{2}\left(1+\frac{\partial X_{t}}{\partial X_{d}}\right)$ y si a los términos entre paréntesis restamos y sumamos $\frac{\partial X_{t}}{\partial X_{d}} \frac{X_{d}}{X_{t}}$, tenemos entonces $S_{d}^{2}\left[1-\frac{\partial X_{t}}{\partial X_{d}} \frac{X_{d}}{X_{t}}+\frac{\partial X_{t}}{\partial X_{d}} \frac{X_{d}}{X_{t}}+\frac{\partial X_{t}}{\partial X_{d}}\right]$, lo anterior tampoco cambia si multiplicamos y dividimos el último término por $X_{t}$, así $S^{2}{ }_{d}\left[1-\frac{\partial X_{t}}{\partial X_{d}} \frac{X_{d}}{X_{t}}+\frac{\partial X_{t}}{\partial X_{d}} \frac{X_{d}}{X_{t}}+\frac{\partial X_{t}}{\partial X_{d}} \frac{X_{t}}{X_{t}}\right]$ Factorizando los dos últimos términos, entonces: $S^{2}{ }_{d}\left[1-\frac{\partial X_{t}}{\partial X_{d}} \frac{X_{d}}{X_{t}}+\frac{\partial X_{t}}{\partial X_{d}}\left(\frac{X_{d}}{X_{t}}+\frac{X_{t}}{X_{t}}\right)\right]$. Pero, como $X_{d}+X_{t}=X$, por ello la anterior expresión es $S^{2}{ }_{d}\left[1-\frac{\partial X_{t}}{\partial X_{d}} \frac{X_{d}}{X_{t}}+\frac{\partial X_{t}}{\partial X_{d}} \frac{X}{X_{t}}\right]$. Multiplicamosahora elcorchete, tenemos $S^{2}{ }_{d}\left(1-\frac{\partial X_{t}}{\partial X_{d}} \frac{X_{d}}{X_{t}}\right)+S^{2}{ }_{d} \frac{\partial X_{t}}{\partial X_{d}} \frac{X}{X_{t}}$.

Pero como $S_{d}=\frac{X_{d}}{X}$, el último sumando es igual a $\left(\frac{X_{d}}{X}\right)^{2} \frac{\partial X_{t}}{\partial X_{d}} \frac{X}{X_{t}} ;$ es decir $\frac{X_{d}}{X} \frac{\partial X_{t}}{\partial X_{d}} \frac{X_{d}}{X_{t}}$, o de igual manera $S_{d} \frac{\partial X_{t}}{\partial X_{d}} \frac{\partial X_{d}}{X_{t}}$.

Así entonces: $S_{d}^{2}\left(1-\frac{\partial X_{t}}{\partial X_{d}} \frac{X_{d}}{X_{t}}\right)+S_{d} \frac{\partial X_{t}}{\partial X_{d}} \frac{\partial X_{d}}{X_{t}}$ es: $S_{d}^{2}\left(1-\varepsilon_{d t}\right)+S_{d} \varepsilon_{d t}$

Por lo tanto, queda demostrado que $B=S_{d}^{2}\left(1-\varepsilon_{d t}\right)+S_{d} \varepsilon_{d t}$; con $S_{d}=\sum_{i=1}^{d} S_{i}$, como índice de concentración de mercado de las empresas dominantes.

Los modelos desarrollados en este primer punto muestran que el índice de Lerner, o margen precio-costos marginal, está estructuralmente relacionados con los índices de concentración ( $H$ y $\left.C_{R d}\right)$.

En la aplicación de estos modelos a sectores industriales de México, economía emergente como la colombiana, y en la que se utilizó datos contables, se encontró una correlación positiva 
y significativa entre los índices de concentración $C_{4}$ y $H$, y el desempeño industrial ${ }^{6}$.

La validación empírica de los modelos en la economía colombiana utilizaría datos de corte transversal obtenidos de los estados financieros publicados según sectores industriales por la Superintendencia de Sociedades, sección estados financieros (SIREM)

\section{Determinación del índice de LeRner en un MOdelo de OLIGOPOLIO CON FINANCIACIÓN EXTERNA ${ }^{7}$}

Partimos de los siguientes supuestos y definiciones. Sea $i$ una empresa que opera en un mercado bajo oligopolio de un producto homogéneo y financia su inversión acudiendo al mercado (imperfecto) de capital (financia la inversión con la venta de bonos), por lo que existe la posibilidad de quiebra o bancarrota.

Para ello definimos las siguientes variables:

$K_{E}=$ Es el capital inicial aportado por los socios y heredado del período anterior

$I=$ La inversión corriente financiada con la venta de bonos, cada uno de ellos a una unidad monetaria.

$B=$ Bonos

$K_{i}=$ Capital de la empresa $i$

$P_{0}{ }^{K}=$ Precio inicial de los activos de capital

El capital de la empresa $i$ al inicio del período es $K_{i}=K_{E}+I$; y su valor es $P_{0}{ }^{K} K_{i}=P_{0}{ }^{K} K_{E}+P_{0} I$. Pero como el valor de la inversión corriente se financia con bonos, entonces $P_{0}{ }^{K} I_{i}=B$, por lo tanto: $P_{0}{ }^{K}$ $K_{i}=P_{0}{ }^{K} K_{E}+B$, lo que equivale a tener que $P_{0}{ }^{K} K_{E}=P_{0}{ }^{K} K_{i}-B$ (1)

6 FERNÁNDEZ TORRES, Alfredo (2006). El Desempeño Industrial como Medida Indirecta del Poder de Mercado. En: Revista Comercio Exterior, BANCOMEX, Vol. 56 No. 1, p. 47

7 Este desarrollo se basa en Gracia (1996) 
Si no hay bancarrota la empresa redime todos los bonos al final del período pagando $(1+r)$ unidades monetarias por cada bono, siendo $r$ la tasa de interés de los bonos. Si definimos además:

$\delta=$ Tasa de depreciación de los activos de capital

$(1-\delta)=$ Disposición de capital para el período siguiente

$r^{*} \quad=$ Tasa de interés sobre activos seguros

El valor presente descontado (valor de la empresa neto de capital inicial) de la empresa $i$ es:

$$
V P D_{i}=\frac{P Q_{i}+\left(1-\delta_{i}\right) P_{1}^{K} K_{i}-W_{i} L_{i}-\left(1+r_{i}\right) B_{i}+\theta_{i}}{\left(1+r^{*}\right)}-P_{0}{ }^{K} K_{E i}
$$

En la que:

$P_{1}{ }^{K}=$ Precio de los activos de capital al final del período

$P Q_{i}=$ Valor de la producción de la empresa $i$

$W_{i} L_{i}=$ Costo del trabajo

$\theta=$ Variable aleatoria que refleja la incertidumbre inicial sobre la situación de la empresa al final del período

$\left(1+r_{i}\right) B=\quad$ Pago por rendimiento de bonos (pago de capital más intereses)

(1- $\left.\delta_{i}\right) P_{1}{ }^{K} K_{i}=$ Valor de los activos de la empresa al final del período (se descuenta depreciación)

Si sustituimos la ecuación (1) en (2) obtenemos:

$$
\begin{gathered}
V P D_{i}=\frac{P Q_{i}+\left(1-\delta_{i}\right) P_{1}^{K} K_{i}-W_{i} L_{i}-\left(1+r_{i}\right) B_{i}+\theta_{i}}{\left(1+r^{*}\right)}-\left(P_{0}{ }^{K} K_{i}-B_{i}\right) \text {, es decir } \\
V P D_{i}=\frac{P Q_{i}+\left(1-\delta_{i}\right) P_{1}{ }^{K} K_{i}-W_{i} L_{i}-\left(1+r_{i}\right) B_{i}+\theta_{i}}{\left(1+r^{*}\right)}-P_{0}{ }^{K} K_{i}+B_{i} \text {, o también } \\
V P D_{i}=\frac{P Q_{i}+\left(1-\delta_{i}\right) P_{1}^{K} K_{i}-W_{i} L_{i}-\left(1+r_{i}\right) B_{i}+\theta_{i}-\left(1+r^{*}\right) P_{0}{ }^{K} K_{i}+\left(1+r^{*}\right) B_{i}}{\left(1+r^{*}\right)}
\end{gathered}
$$


Reordenando la expresión anterior, tenemos:

$$
V P D_{i}=\frac{P Q_{i}+\left(1-\delta_{i}\right) P_{1}^{K} K_{i}-\left(1+r^{*}\right) P_{0}^{K} K_{i}-W_{i} L_{i}-\left(1+r_{i}\right) B_{i}+\left(1+r^{*}\right) B_{i}+\theta_{i}}{\left(1+r^{*}\right)}
$$

En esta última ecuación, la expresión $-\left(1+r_{i}\right) B_{i}+\left(1+r^{*}\right) B_{i}$ se reduce a: $-B_{i}-r_{i} B_{i}+B_{i}+r^{*} B_{i}$, es decir $-r_{i} B_{i}+r^{*} B_{i}$, que al tomar factor común $B_{i}$ obtenemos - $\left(r-r^{*}\right) B_{i}$.

Igualmente, la expresión $\left(1-\delta_{i}\right) P_{1}{ }^{K} K_{i}-\left(1+r^{*}\right) P_{0}{ }^{K} K_{i}$ se reduce

Tomando factor común $K_{i}$ tenemos $\left[\left(1-\delta_{i}\right) P_{1}^{K}-\left(1+r^{*}\right) P_{0}{ }^{K}\right] K_{i}$. Multiplicar y dividir por $\frac{P_{0}{ }^{K}}{P_{0}{ }^{K}}$, entonces $\left[\left(1-\delta_{i}\right) P_{1}^{K}-\left(1+r^{*}\right) P_{0}{ }^{K}\right] \frac{P_{0}{ }^{K}}{P_{0}{ }^{K}} * K_{i}$. Y lo anterior es igual a $\left[\left(1-\delta_{i}\right) \frac{P_{1}{ }^{K}}{P_{0}{ }^{K}}-\left(1+r^{*}\right) \frac{P_{0}{ }^{K}}{P_{0}{ }^{K}}\right] P_{0}{ }^{K} K_{i}$, o sea $\left[\left(1-\delta_{i}\right) \frac{P_{1}^{K}}{P_{0}{ }^{K}}-1-r^{*}\right] P_{0}{ }^{K} K_{i}$;

Si a esta última expresión le sumamos y restamos $\delta_{i}$, tenemos $\left[-r^{*}-\delta_{i}+\left(1-\delta_{i}\right) \frac{P_{1}{ }^{K}}{P_{0}{ }^{K}}-1+\delta_{i}\right] P_{0}{ }^{K} K_{i}$, y es igual a $\left[-r^{*}-\delta_{i}+\left(1-\delta_{i}\right) \frac{P_{1}^{K}}{P_{0}{ }^{K}}-\left(1-\delta_{i}\right)\right] P_{0}{ }^{K} K_{i}$.

Tomando factor común $\left(1-\delta_{i}\right)$, obtenemos $\left[-r^{*}-\delta_{i}+\left(1-\delta_{i}\right)\left(\frac{P_{1}{ }^{K}}{P_{0}{ }^{K}}-1\right)\right] P_{0}{ }^{K} K_{i}$, o lo que es lo mismo $\left[-r^{\cdot}-\delta_{i}+\left(1-\delta_{i}\right)\left(\frac{P_{1}{ }^{K}-P_{0}{ }^{K}}{P_{0}{ }^{K}}\right)\right] P_{0}{ }^{K} K_{i}$, o también igual a $-\left[r^{*}+\delta_{i}-\left(1-\delta_{i}\right)\left(\frac{P_{1}^{K}-P_{0}{ }^{K}}{P_{0}{ }^{K}}\right)\right] P_{0}{ }^{K} K_{i} \cdot$ 
Si hacemos que $\lambda_{i}=r^{*}+\delta_{i}-\left(1-\delta_{i}\right) \frac{P_{1}{ }^{K}-P_{0}{ }^{K}}{P_{0}{ }}$, entonces el valor presente descontado, o valor de la empresa neto de capital, es: $V P D_{i}=\frac{\left(P Q_{i}-\lambda_{i} P_{0}{ }^{K} K_{i}-W_{i} L_{i}\right)+\theta_{i}-\left(r_{i}-r^{*}\right) B_{i}}{1+r^{*}}$ y en la que:

$P Q_{i}-\lambda_{i} P_{0}{ }^{K} K_{i}-W_{i} L_{i}=$ Es el beneficio económico bruto

$\theta_{i}=$ La incertidumbre inicial sobre la situación final de la empresa

$\left(r_{i}-r^{*}\right) B_{i}=$ Es el costo de oportunidad de financiarse con emisión de bonos

Si la empresa al final del período no es capaz de redimir los bonos, entrará en bancarrota.

\subsection{El valor presente descontado de venta de la empresa $\left(V P D V_{i}\right)$}

Se define como la suma del valor presente descontado de la empresa y el valor del capital inicial aportado por los socios, esto es $V P D V_{i}=V P D_{i}+P_{0}{ }^{K} K_{E i}$, y es igual a

$$
V P D V_{i}=\frac{\left(P Q_{i}-\lambda_{i} P_{0}{ }^{K} K_{i}-W_{i} L_{i}\right)+\theta_{i}-\left(r_{i}-r^{*}\right) B_{i}}{1+r^{*}}+P_{0}{ }^{K} K_{E i},
$$

o lo que es lo mismo:

$$
V P D V_{i}=\frac{\left(P Q_{i}-\lambda_{i} P_{0}^{K} K_{i}-W_{i} L_{i}\right)+\theta_{i}-\left(r_{i}-r^{*}\right) B_{i}+\left(1+r^{*}\right) P_{0}{ }^{K} K_{E i}}{1+r^{*}}
$$

Sea ahora $\theta^{*}$ el valor de $\theta$ que hace exactamente al valor presente descontado de venta igual a cero (o sea $V P D V_{i}=0$ ); y sea además $f(\theta)$ la función de densidad asignada por los propietarios a $\theta$, pues recordemos que es una variable aleatoria que refleja la incertidumbre inicial. Si tenemos $\theta^{*}$ tal que $V P D V_{i}=0$, entonces:

$$
0=\frac{\left(P Q_{i}-\lambda_{i} P_{0}{ }^{K} K_{i}-W_{i} L_{i}\right)+\theta_{i}^{*}-\left(r_{i}-r^{*}\right) B_{i}+\left(1+r^{*}\right) P_{0}^{K} K_{E i}}{\left(i+r^{*}\right)} \text {, o también }
$$




$$
\begin{aligned}
& 0=\left(P Q_{i}-\lambda_{i} P_{0}{ }^{K} K_{i}-W_{i} L_{i}\right)+\theta_{i}^{*}-\left(r_{i}-r^{*}\right) B_{i}+\left(1+r^{*}\right) P_{0}^{K} K_{E i} \text {, por lo que } \\
& \theta_{i}^{*}=\left(r_{i}-r^{*}\right) B_{i}-\left(P Q_{i}-\lambda_{i} P_{0}^{K} K_{i}-W_{i} L_{i}\right)-\left(1+r^{*}\right) P_{0}^{K} K_{E i} \text {, y que tiene las }
\end{aligned}
$$
siguientes funciones de densidad de probabilidad asignadas por los propietarios:

$P_{r}(b)=$ Probabilidad de bancarrota

$P_{r}(\bar{b})=$ Probabilidad de no bancarrota

$\mathrm{E}(\theta \mid \bar{b})=$ Valor esperado de $\theta$ condicionado a no bancarrota

$$
\begin{aligned}
& P_{r}(b)=P_{r}\left(\theta \leq \theta^{*}\right) \int_{-\infty}^{\theta^{*}} f(\theta) \partial \theta \\
& P_{r}(\bar{b})=P_{r}\left(\theta \geq \theta^{*}\right) \int_{\theta^{*}}^{\infty^{+}} f(\theta) \partial \theta \\
& E(\theta \mid \bar{b})=\int_{\theta^{*}}^{\infty+} \theta f(\theta) \partial \theta^{18}
\end{aligned}
$$

\subsection{Maximización del valor presente esperado de la empresa}

Los empresarios maximizan el valor presente esperado de la empresa sujeto a la financiación de la inversión mediante la emisión de bonos. El problema es:

$$
\operatorname{Max} E\left(V P D_{i}\right)=P_{0}{ }^{K} K_{E i} P(b)+\frac{\left[P Q_{i}-W_{i} L_{i}-\lambda_{i} P_{0}{ }^{K} K_{i}-\left(r_{i}-r^{*}\right)\right] P(\bar{b})+E(\theta \mid \bar{b})}{\left(1+r^{*}\right)}
$$

Sujeto a:

$$
B_{i}=P_{0}^{K} I_{i}=P_{0}^{K} K_{i}-P_{0}^{K} K_{E i}
$$

Utilizando el método del multiplicador de Lagrange, tenemos que maximizar la siguiente función:

$$
\begin{aligned}
& \underset{(L, K, B)}{\operatorname{Max} \ell}=-P_{0}{ }^{K} K_{E i} P(b)+\left[P Q_{i}-W_{i} L_{i}-\lambda_{i} P_{0}{ }^{K} K_{i}-\left(r_{i}-r^{*}\right) B_{i}\right] P(\bar{b})+E(\theta \mid \bar{b})+ \\
& \ell\left(B_{i}-P_{0}{ }^{K} K_{i}+P_{0}{ }^{K} K_{E i}\right)
\end{aligned}
$$

8 Ver GRACIAS (1996) y (1999) 
Las derivadas parciales, igualadas a cero, respecto a $L_{i}, K_{i}, B_{i}$ son:

- $\frac{\partial \ell}{\partial L_{i}}=0$, es $\frac{\partial Q_{i}}{\partial L_{i}} I M g_{i}=W_{i}+\frac{\partial r_{i}}{\partial Q_{i}} \frac{\partial Q_{i}}{\partial L_{i}} B_{i}$. Si multiplicamos y dividimos por $\frac{Q_{i}}{r_{i}} \mathrm{a} \frac{\partial r_{i}}{\partial Q_{i}} \frac{\partial Q_{i}}{\partial L_{i}} B_{i}$, obtenemos $\frac{\partial Q_{i}}{\partial L_{i}} I M g_{i}=W_{i}+\frac{\partial r_{i}}{\partial Q_{i}} \frac{Q_{i}}{r_{i}} \frac{r_{i}}{Q_{i}} B_{i} \frac{\partial Q_{i}}{\partial L_{i}}$ o sea $\frac{\partial Q_{i}}{\partial L_{i}} I M g_{i}=W_{i}+\varepsilon_{r Q i} \frac{r_{i} B_{i}}{Q_{i}} \frac{\partial Q_{i}}{\partial L_{i}} ; \operatorname{con} \varepsilon_{r Q i} \frac{\partial r_{i}}{\partial Q_{i}} \frac{Q_{i}}{r_{i}}$.

- $\frac{\partial \ell}{\partial K_{i}}=0$, es $\frac{\partial Q_{i}}{\partial K_{i}} I M g_{i}=\lambda_{i} P_{0}{ }^{K}+\frac{\partial r_{i}}{\partial Q_{i}} \frac{\partial Q_{i}}{\partial K_{i}} B_{i}+\ell\left(1+r^{*}\right) P_{0}{ }^{K}$. Si como en el caso anterior multiplicamos y dividimos por $\frac{Q_{i}}{r_{i}}$ a los mismos términos y además tomamos factor común $P_{0}{ }^{K}$, tenemos que $\frac{\partial Q_{i}}{\partial K_{i}} I M g_{i}=\left[\lambda_{i}+\ell\left(1+r^{*}\right)\right] P_{0}{ }^{K}+\frac{\partial r_{i}}{\partial Q_{i}} \frac{Q_{i}}{r_{i}} \frac{r_{i} B_{i}}{Q_{i}} \frac{\partial Q_{i}}{\partial K_{i}}$ es decir, $\frac{\partial Q_{i}}{\partial K_{i}} I M g_{i}=\left[\lambda_{i}+\ell\left(1+r^{*}\right)\right] P_{0}^{K}+\varepsilon_{r Q i} \frac{r_{i} B_{i}}{Q_{i}} \frac{\partial Q_{i}}{\partial K_{i}}$.

- $\frac{\partial \ell}{\partial B_{i}}=0$, es $\frac{-\left(r_{i}-r^{*}\right)-B_{i} \frac{\partial r_{i}}{\partial B_{i}}}{\left(1+r^{*}\right)}=-\ell$, o también $-r_{i}+r^{*}-B_{i} \frac{\partial r_{i}}{\partial B_{i}}=-\ell\left(1+r^{*}\right)$ es decir $r_{i}-r^{*}+B_{i} \frac{\partial r_{i}}{\partial B_{i}}=\ell\left(1+r^{*}\right)$. Si multiplicamos y dividimos a $B_{i} \frac{\partial r_{i}}{\partial B_{i}}$ por $r_{i}$, tenemos $r_{i}-r^{*}+B_{i} \frac{\partial r_{i}}{\partial B_{i}} \frac{r_{i}}{r_{i}}=\ell\left(1+r^{*}\right)$ o sea $r_{i}-r^{*}+\frac{B_{i}}{r_{i}} \frac{\partial r_{i}}{\partial B_{i}} r_{i}=\ell\left(1+r^{*}\right)$ y si $\varepsilon_{r B i}=\frac{\partial_{r i}}{\partial_{B i}} \frac{B_{i}}{r_{i}}$, entonces tenemos que $r_{i}-r^{*}+\varepsilon_{r B i} r_{i}=\ell\left(1+r^{*}\right)$.

Con:

$\varepsilon_{r Q i}: \quad$ Elasticidad de tasa de interés de los bonos respecto al volumen de producción. 
$\varepsilon_{r B r}: \quad$ Elasticidad de la tasa de interés de los bonos respecto a la cantidad de bonos.

$I M g_{i}$ : Ingreso marginal de la empresa $i$.

$$
\begin{gathered}
\text { Ahora, como } \frac{\partial Q_{i}}{\partial K_{i}} I M g_{i}=\left[\lambda_{i}+\ell\left(1+r^{*}\right)\right] p_{0}{ }^{k}+\varepsilon_{r Q i} \frac{r_{i} B_{i}}{Q_{i}} \frac{\partial Q_{i}}{\partial K_{i}}, \text { con } \\
\ell\left(1+r^{*}\right)=r_{i}-r^{*}+\varepsilon_{r B i} r_{i} \text { y } \lambda_{i}=r^{*}+\delta_{i}-\left(1-\delta_{i}\right) \frac{P_{1}^{k}-P_{0}{ }^{k}}{P_{0}{ }^{k}} \text {, entonces: } \\
\frac{\partial Q_{i}}{\partial K_{i}} I M g_{i}=\left[r^{*}+\delta_{i}-\left(1-\delta_{i}\right) \frac{P_{1}^{k}-P_{0}{ }^{k}}{P_{0}{ }^{k}}+r_{i}-r^{*}+\varepsilon_{r B i} r_{i}\right] P_{0}{ }^{k}+\varepsilon_{r Q i} \frac{r_{i} B_{i}}{Q_{i}} \frac{\partial Q_{i}}{\partial k_{i}} .
\end{gathered}
$$

Si cancelamos $r^{*}$ y tomamos factor común $r_{i}$ entonces:

$$
\begin{aligned}
& \frac{\partial Q_{i}}{\partial K_{i}} I M g_{i}=\left[\delta_{i}-\left(1-\delta_{i}\right) \frac{P_{1}^{k}-P_{0}{ }^{k}}{P_{0}{ }^{k}}+\left(1+\varepsilon_{r B i}\right) r_{i}\right] P_{0}{ }^{k}+\varepsilon_{r Q i} \frac{r_{i} B_{i}}{Q_{i}} \frac{\partial Q_{i}}{\partial k_{i}} . \\
& \text { Si } \lambda_{B i}=\left[\delta_{i}-\left(1-\delta_{i}\right) \frac{P_{1}^{k}-P_{0}{ }^{k}}{P_{0}{ }^{k}}+\left(1+\varepsilon_{r B i}\right) r_{i}\right], \text { entonces } \\
& \frac{\partial Q_{i}}{\partial K_{i}} I M g_{i}=\lambda_{B i} P_{0}^{k}+\varepsilon_{r Q i} \frac{r_{i} B_{i}}{Q_{i}} \frac{\partial Q_{i}}{\partial k_{i}} .
\end{aligned}
$$

\subsubsection{Productividad Marginal del trabajo}

$$
\operatorname{Si} \frac{\partial Q_{i}}{\partial L_{i}} I M g_{i}=W_{i}+\varepsilon_{r Q i} \frac{r_{i} B_{i}}{Q_{i}} * \frac{\partial Q_{i}}{\partial L_{i}} \text {, al despejar el producto marginal }
$$

del trabajo $\left(\frac{\partial Q_{i}}{\partial L_{i}}\right)$, tenemos: $\frac{\partial Q_{i}}{\partial L_{i}} I M g_{i}-\varepsilon_{r Q i} \frac{r_{i} B_{i}}{Q_{i}} * \frac{\partial Q_{i}}{\partial L_{i}}=W_{i}$, o sea $\frac{\partial Q_{i}}{\partial L_{i}}\left(I M g_{i}-\varepsilon_{r Q i} \frac{r_{i} B_{i}}{Q_{i}}\right)=W_{i}$ 


$$
\text { Es decir: } \frac{\partial Q_{i}}{\partial L_{i}}=\frac{W_{i}}{I M g_{i}-\varepsilon_{r Q i} \frac{r_{i} B_{i}}{Q_{i}}}
$$

\subsubsection{Producto Marginal del Capital}

Como $\frac{\partial Q_{i}}{\partial K_{i}} I M g_{i}=\lambda_{B i} P_{0}^{K}+\varepsilon_{r Q i} \frac{r_{i} B_{i}}{Q_{i}} * \frac{\partial Q_{i}}{\partial K_{i}}$, tenemos que:

$\frac{\partial Q_{i}}{\partial K_{i}} I M g_{i}-\varepsilon_{r Q_{i}} \frac{r_{i} B_{i}}{Q_{i}} \frac{\partial Q_{i}}{\partial K_{i}}=\lambda_{B i} P_{0}^{K}$, por ello el producto marginal de capital es: $\frac{\partial Q_{i}}{\partial K_{i}}=\frac{\lambda_{B i} P_{0}^{K}}{I M g_{i}-\varepsilon_{r Q_{i}} \frac{r_{i} B_{i}}{Q_{i}} \frac{\partial Q_{i}}{\partial K_{i}}}$

\subsection{La expresión para el margen del precio sobre el costo medio de producción a largo plazo}

Supongamos que la empresa tiene una función de producción $Q_{i}=Q\left(L_{i}, K_{i}\right)$, localmente homogénea, en un entorno $Q_{i}$, la relación entre costo medio y marginal (índice de rendimientos a escala) es

$$
F C_{i}(Q)_{i}=\frac{L_{i} \frac{\partial Q_{i}}{\partial L_{i}}+K_{i} \frac{\partial Q_{i}}{\partial K_{i}}}{Q_{i}} \text {, pero como } \frac{\partial Q_{i}}{\partial L_{i}} \mathrm{y} \frac{\partial Q_{i}}{\partial K_{i}} \text { son las produc- }
$$
tividades marginales del trabajo y el capital, respectivamente, tenemos que la relación $F C_{i}$ es:

$$
\begin{aligned}
& F C_{i}\left(Q_{i}\right)=\frac{L_{i} \frac{W_{i}}{I M g_{i}-\varepsilon_{r Q i} \frac{r_{i} B_{i}}{Q_{i}}}+K_{i} \frac{\lambda_{B i} P_{0^{k}}}{I M g_{i}-\varepsilon_{r Q i} \frac{r_{i} B_{i}}{Q_{i}}}}{Q_{i}} ; \text { lo que es igual a: } \\
& F C_{i}=\frac{W_{i} L_{i}+\lambda_{i} P_{0}^{k} K_{i}}{Q_{i}\left(I M g_{i}-\varepsilon_{r Q i} \frac{r_{i} B_{i}}{Q_{i}}\right)}
\end{aligned}
$$


Ahora, si $P(Q)$ es la función inversa de demanda y el ingreso es $p(Q) Q_{i}$, entonces el ingreso marginal es $I M g_{i}=\frac{\partial P(Q) Q_{i}}{\partial Q_{i}}=P+Q_{i} \frac{\partial P}{\partial Q} \frac{\partial Q}{\partial Q_{i}}$. Si multiplicamos y dividimos por $\frac{Q}{P}$ el lado derecho de la expresión anterior con excepción de $P$ tenemos:

$$
\begin{aligned}
& I M g_{i}=P+Q_{i} \frac{\partial P}{\partial Q} \frac{\partial Q}{\partial Q_{i}} \frac{Q}{P} \frac{P}{Q} ; \text { y reordenando, tenemos: } \\
& I M g_{i}=P+\frac{Q_{i}}{Q} \frac{\partial P}{\partial Q} \frac{Q}{P} P .
\end{aligned}
$$

Ahora, $\frac{\partial P}{\partial Q} \frac{Q}{P}$ es la inversa de la elasticidad de la demanda cuyo valor es negativo; por ello $\frac{\partial P}{\partial Q} \frac{Q}{P}=-\frac{1}{\varepsilon_{P X}}$. Además, $\frac{Q_{i}}{Q}$ es la participación en el mercado de la empresa $i$, o sea $S_{i}$. De esta manera, $I M g_{i}=P-\frac{p}{\varepsilon_{P X}} S_{i} \frac{\partial Q}{\partial Q_{i}}$ o también $P\left(1-\frac{1}{\varepsilon_{P X}} S_{i} \frac{\partial Q}{\partial Q_{i}}\right)$, siendo $\frac{\partial Q}{\partial Q_{i}}$ la variación conjetural.

Si $Q=Q_{i}+Q_{j}$, haciendo a $Q_{j}$ como la producción del resto de las empresas de la industria en el mercado en oligopolio (no incluye la empresa $i$ ), también es cierto que $Q_{j}=Q-Q_{i}$.

Ahora, $\frac{\partial Q}{\partial Q_{i}}$ es entonces $\frac{\partial Q_{i}}{\partial Q_{i}}+\frac{\partial Q_{j}}{\partial Q_{i}}$, es decir $\frac{\partial Q}{\partial Q_{i}}=\left(1+\frac{\partial Q_{j}}{\partial Q_{i}}\right)$.

Si multiplicamos y dividimos a la variación conjetural $\frac{\partial Q_{j}}{\partial Q_{i}}$ por $\frac{Q_{i}}{Q_{j}}$ y definimos a la elasticidad de la variación conjetural como $\alpha_{i}=\frac{\partial Q_{j}}{\partial Q_{i}} \frac{Q_{i}}{Q_{j}}$, tenemos que $\frac{\partial Q}{\partial Q_{j}}=\left(1+\frac{\partial Q_{j}}{\partial Q_{i}} \frac{Q_{i}}{Q_{j}} \frac{Q_{j}}{Q_{i}}\right)$, es también $\frac{\partial Q}{\partial Q_{j}}=\left(1+\alpha_{i} \frac{Q_{j}}{Q_{i}}\right)$. Ahora, $Q_{j}=Q-Q_{i}$, entonces $\frac{\partial Q}{\partial Q_{j}}=\left[1+\alpha_{i}\left(\frac{Q_{j}}{Q_{i}}-\frac{Q_{i}}{Q_{j}}\right)\right] ;$ es decir, y siendo $\frac{Q}{Q_{i}}$ el inverso de $S_{i}$ (la participación en el mercado 
de la empresa $i)$, que $\frac{\partial Q}{\partial Q_{j}}=\left[1+\alpha_{i}\left(\frac{1}{S_{i}}-1\right)\right]$. Si reemplazamos lo anterior en la fórmula para el ingreso marginal tenemos entonces: $I M g_{i}=P\left[1-\frac{1}{\varepsilon_{P X}} S_{i}\left[1+\alpha_{i}\left(\frac{1}{S_{i}}-1\right)\right]\right] ;$ y simplificado tenemos $I M g_{i}=P\left[1-\frac{S_{i}\left(1+\frac{\alpha_{i}}{S_{i}}-\alpha_{i}\right)}{\varepsilon_{P X}}\right]$, es decir: $I M g_{i}=P\left[1-\frac{S_{i}+\alpha_{i}-\alpha_{i} S_{i}}{\varepsilon_{P X}}\right]$, tomando factor común $S_{i}$, entonces $I M g_{i}=P\left[1-\frac{\alpha_{i}+\left(1-\alpha_{i}\right) S_{i}}{\varepsilon_{P X}}\right]$, es la ecuación
para el ingreso marginal

Si reemplazamos esta ecuación del ingreso marginal en la ecuación $F C_{i}\left(Q_{i}\right)$, se deduce la ecuación para el margen precio sobre los costos medio de producción a largo plazos como se sigue:

$$
\begin{aligned}
& F C_{i}=\frac{W_{i} L_{i}+\lambda_{B i} P_{0}^{k} K_{i}}{Q_{i}\left[P *\left(\frac{1-\alpha_{i}+\left(1-\alpha_{i}\right) S_{i}}{\varepsilon_{P X}}\right)-\varepsilon_{r Q i} \frac{r_{i} B_{i}}{Q_{i}}\right]} \text {. Lo anterior equivale a: } \\
& F C_{i}\left[Q_{i} P\left(1-\frac{\alpha_{i}+\left(1-\alpha_{i}\right) S_{i}}{\varepsilon_{P X}}\right)-\varepsilon_{r Q i} \frac{r_{i} B_{i}}{Q_{i}} Q_{i}\right]=W_{i} L_{i}+\lambda_{B i} P_{0}^{k} K_{i} \text {, tomando }
\end{aligned}
$$

factor común $P Q_{i}$ en el lado izquierdo de la expresión y multiplicando por $F C_{i}$ tenemos:

$$
P Q_{i}\left[\left(1-\frac{\alpha_{i}+\left(1-\alpha_{i}\right) S_{i}}{\varepsilon_{P X}}\right) F C_{i}-F C_{i} \varepsilon_{e Q i} \frac{r_{i} B_{i}}{P Q_{i}}\right]=W_{i} L_{i}+\lambda_{B i} P_{0}^{k} K_{i} \text {, restemos }
$$

ahora a $P Q_{i}$ en cada lado de la igualdad, entonces:

$$
P Q_{i}-P Q_{i}\left[\left(1-\frac{\alpha_{i}+\left(1-\alpha_{i}\right) S_{i}}{\varepsilon_{P X}}\right) F C_{i}-F C_{i} \varepsilon_{r Q i} \frac{r_{i} B_{i}}{P Q_{i}}\right]=P Q_{i}-W_{i} L_{i}+\lambda_{B i} P_{0}^{k} K_{i .}
$$

Tomando factor común en lado izquierdo y pasándolo a dividir: 
$1-\left[\left(1-\frac{\alpha_{i}+\left(1-\alpha_{i}\right)}{\varepsilon_{P X}} S_{i}\right) F C_{i}-F C_{i} \varepsilon_{r Q i} \frac{r_{i} B_{i}}{P Q_{i}}\right]=\frac{P Q_{i}-W_{i} L_{i}+\lambda_{B i} P_{0}{ }^{K} K_{i}}{P Q_{i}}$, por lo tanto: $1-F C_{i}+F C_{i} \frac{\alpha_{i}}{\varepsilon_{P X}}+F C_{i} \frac{\left(1-\alpha_{i}\right)}{\varepsilon_{P X}} S_{i}+F C_{i} \varepsilon_{r Q i} \frac{r_{i} B_{i}}{P Q_{i}}=\frac{P Q_{i}-W_{i} L_{i}+\lambda_{B i} P_{0}{ }^{K} K_{i}}{P Q_{i}}$, y es la ecuación de comportamiento para el margen precio/costo medio de largo plazo.

En esta ecuación:

$P=\quad$ Precio del producto

$Q=$ Producción

$W L=$ Costo del trabajo

$\lambda_{B}=\quad$ Costo de uso de servicios de capital

$P_{t}^{K}=$ Precio del capital por unidad en el periodo $t$

$K=\quad$ Stock de capital físico

$F C=$ Índice de rendimiento a escala

$\alpha=\quad$ Elasticidad conjetural

$\varepsilon_{P X}=$ Elasticidad precio de la demanda

$S=\quad$ Cuota de mercado

$r=\quad$ Tasa de interés de los bonos

$B=\quad$ Número de bonos a un valor de una unidad monetaria

$\varepsilon_{r Q}=$ Elasticidad tasa de interés al nivel de producción.

En la ecuación anterior aparecen los determinantes clásicos del margen para mercados en oligopolio: rendimientos a escala, elasticidad de la demanda, cuota de mercado, comportamiento empresarial (variación conjetural).

Este modelo ha sido estimado para la economía española durante el período 1983-1990 por Esperanza Gracia9 ${ }^{9}$ utilizando información tomada de la Central de Balances del Banco de España.

9 GRACIA EXPÓSITO, Esperanza (1996). Márgenes y Cuota de Mercado: Un Análisis Econométrico con Datos Individuales. Madrid: Universidad Complutense de Madrid (Tesis Doctoral) 
La estimación es para un panel de 979 empresas manufactureras españolas; y encuentra, como uno de sus resultados, que la variable con mayor impacto sobre los márgenes es la participación o cuota de mercado de la empresa.

\section{Conclusión}

En este artículo se presenta el desarrollo matemático de algunos modelos teóricos para estimar y diagnosticar la existencia de poder de mercado en diferentes estructuras industriales, e inscritos en el paradigma clásico "Estructura - Conducta - Resultados".

En el modelo de oligopolio tipo Cournot, se concluye que el poder de mercado de la empresa $i$ depende de su cuota de mercado (o participación de la empresa en el mercado de la industria), de la elasticidad precio de la demanda del bien $X$ y de la expectativa (conjetura constante) de la empresa $i$ sobre la conducta de las empresas rivales. La ecuación de la relación es $B_{i}=\frac{S_{i}\left(1+\lambda_{i}\right)}{\varepsilon_{P X}}$, siendo $B_{i}$ en margen precio-costo marginal o índice de Lerner. Para el caso de la industria, el poder de mercado se relaciona con el índice de Herfindahl, la elasticidad precios de la demanda y la variación conjetura. La ecuación para el índice de Lerner es $B=\frac{\left(1+\lambda_{i}\right) H}{\varepsilon_{P X}}$.

Adicionalmente, en mercados dominados por un grupo de empresas, el índice de Lerner para el grupo de empresas dominantes es $B_{d}=\frac{S_{d}\left(1+\lambda_{d t}\right)}{\varepsilon_{P X}} ; \mathrm{y}$ el índice de poder de mercado promedio de la industria, ponderando por la participación relativa en el mercado $S_{d}$ es: $B=\frac{S^{2}{ }_{d}\left(1+\lambda_{d t}\right)}{\varepsilon_{P X}}$. Por último, si el grupo dominante considera la variación conjetural constante, entonces $B=\frac{\left(1-\lambda_{d t}\right) S^{2}{ }_{d}+\varepsilon_{d t} S_{d}}{\mathcal{E}_{P X}}$

En estos casos, el desarrollo del modelo muestra que los índices de concentración están estructuralmente relacionados con el índice de Lerner de poder de mercado.

En el modelo de oligopolio de un producto homogéneo con financiación externa, bajo los supuestos de maximización del valor 
esperado de la empresas por parte del empresario y en un contexto de mercados imperfectos de capitales, se obtuvo una ecuación de comportamiento para el margen precios- costo medio de producción de largo plazo. En esta ecuación aparecen los determinantes clásicos del margen en mercado oligopolístico: rendimientos a escala, elasticidad de la demanda, cuota del mercado y variaciones conjeturales (comportamiento).

\section{Biblografía}

Clarke, R. \& Davies, S.W. (1982). Market structure and Price - cost margins. In: Economica, New series, vol. 49, No 195 (aug.), pp. 277-287.

Cowling, K. \& Waterson, M. (1976). Price-Cost Margins and Market Structure. In: Economica, núm. 43, pp. 267-274.

Feinberg, R.M. (1980). The Lerner Index, Concentration, and Measurement of Market Power. In: Southern Journal, vol. 46, No. 4, pp. 1180-1186.

Fernández, A. (2006). El Desempeño Industrial como Medida Indirecta del Poder de Mercado. En: Revista Comercio Exterior, BANCOMEX, Vol. 56 No. 1, pp. 41-47.

Gracia, E. (1996). Márgenes y Cuota de Mercado: Un Análisis Econométrico con Datos Individuales. Madrid: Universidad Complutense de Madrid (Tesis Doctoral).

Gracia, E. (1999). Márgenes y Cuota de Mercado: Un Análisis con un micro panel. Investigaciones económicas. Vol. XXIII. No 3 1999, pp. 393-428. Universidad Complutense de Madrid - Fundación Empresas Públicas.

Huergo, E. (2001). El Diagnóstico del Poder de Mercado En Economía Industrial: Un Revisión de la Literatura Empírica Española. Madrid: Universidad Complutense de Madrid (Disponible en www.ucm.es/BUCH/cee/ doc/01-12/0112.pdf).

Mazón, C. (2002). El Margen Precio - Costo Marginal en la Encuesta Industrial: 1978-1988. BANCO DE ESPAÑA. Documento de trabajo No. 9205.

Mazón, C. (2002). Márgenes de beneficio, eficiencia y poder de mercado en las empresas españolas. BANCO DE ESPAÑA. Documento de trabajo No. 9204.

Morrison, C.J. (1990). Market Power, Economic Profitability and Productivity Growth Measurement: An Integrated Structural Approach. National Bureau of Economic Research. Working Paper No. 3355. 
Pavitt, K. (1984). Sectoral Patterns of Technical Change: Towards a taxonomy and theory. In: Science Policy Research 13 pp. 343-373.

Sawhney, P.K. \& Sawhney, B.L. (1973) Capacity-Utilization, Concentration, and Price-Cost Margins: results on indian industries. In: The Journal of Industrial Economics, vol. XX1, No. 2 (April).

Shapiro, M.D. (1987) Measuring Market Power in U.S. industry. National Bureau of Economic Research. Working Paper No. 2212.

Shepherd, W.G. (1972). The Elements of Market Structure. In: The Review of Eonomics, vol.54, No1 (Feb.) pp25-37. 